\title{
Gain Enhancement Techniques for mm-Wave On- Chip Antennas on Lossy CMOS Platforms
}

\author{
Atif Shamim and Haoran Zhang \\ Electrical Engineering Program, CEMSE Division \\ King Abdullah University of Science and Technology \\ Thuwal, Saudi Arabia \\ Atif.shamim@kaust.edu.sa; Haoran.zhang@kaust.edu.sa
}

\begin{abstract}
With the advent of number of applications in the mm-wave bands, antenna sizes are becoming small enough to fit them on mm-scale Integrated Circuits (ICs), commonly known as microchips. Integration of antenna on a chip along with the driving circuits in the standard CMOS process can enable a true RF System-on-Chip (SoC) solution, however, Silicon used in CMOS is very lossy and has a high dielectric constant. This results in low gain and efficiency antennas which also suffer from surface waves issues at $\mathbf{m m}$-wave frequencies. In this paper, we will present Artificial Magnetic Conductor (AMC) surface to increase the gain/efficiency of the on-chip antenna realized in standard CMOS platforms. The gain and radiation efficiency of $1.7 \mathrm{dBi}$ and $42.52 \%$ are achieved respectively.
\end{abstract}

Keywords-mm-wave, System-on-chip, gain, efficiency, AMC, on-chip antenna

\section{INTRODUCTION}

Recently, there are a lot of research interests in the mmwave frequency bands which are eligible for the applications that require high data rate, high resolution, and low interference. Besides, the sizes of mm-wave frequency antenna are small enough to fit on millimeter-scale Integrated Circuits which made it possible to integrated on-chip antenna and RF modules with standard CMOS process in one single microchip as a real RF SoC solution.

However, the main drawbacks of standalone on-chip antenna are its low radiation efficiency and gain because the silicon substrate used in standard CMOS process has low resistivity $(\rho=10 \Omega \cdot \mathrm{cm})$ and high relative permittivity $\left(\varepsilon_{r}=11.9\right)$ which causes the most RF power would be absorbed by the lossy silicon substrate rather than being radiated by the on-chip antenna. As a result, the radiation efficiency and gain decrease to a low level. Besides, the on-chip antenna also suffers from surface wave issues. The typical thickness of silicon substrate in standard CMOS process is 300-500um which is electrically thick compared with the wavelength of mm-wave, making it easier to excite surface waves [1].

Several methods have been reported in previous work for gain enhancement and efficiency enhancement of the on-chip antenna. Quartz superstrate is proposed to improve the radiation performance at the boresight [2]. Silicon micromatching technology is investigated to isolate the on-chip antenna from the lossy substrate [3]. The silicon lens has been used to change the undesired surface waves into objective radiation [4]. However, all the methods require extra fabrication process, making them incompatible with the standard CMOS process. In this paper, Artificial Magnetic Conductor (AMC) is studied for the gain enhancement of a $77 \mathrm{GHz}$ on-chip monopole antenna in standard $0.18 \mu \mathrm{m}$ CMOS process.

\section{REFLECTING SURFACES FOR ON-CHIP ANTENNA}

The standard CMOS technology typically contains $5-10 \mu \mathrm{m}$ thick $\mathrm{SiO}_{2}$ layer embedding with 6 to 9 metal layers. Since the top metal layer is used for designing the on-chip antenna, in order to improve the radiation performance at the boresight, the bottom metal layer (M1) could be used as Perfect Electrical Conductor (PEC) ground plane to isolate the on-chip antenna from the lossy silicon substrate. However, the on-chip antenna must be implemented as a planar antenna on the top metal layer which is electrically close to the ground plane on M1 layer, so the radiation generated by the induced image current on the ground plane would cancel with the on-chip antenna's radiation, causing the low gain and radiation efficiency.

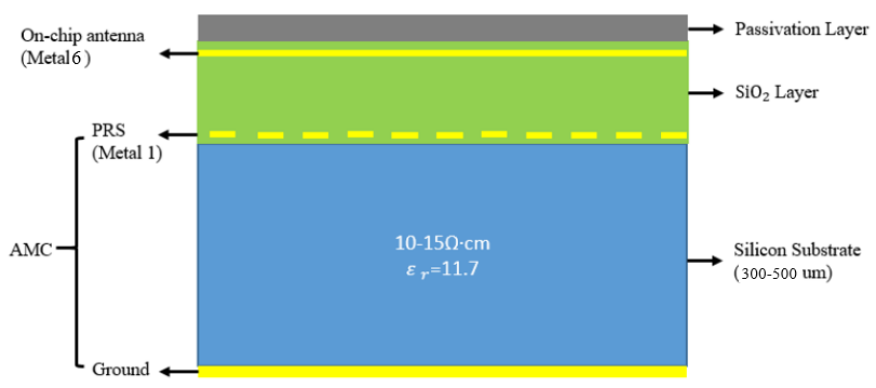

Fig. 1. Cross-section view of AMC in CMOS stack-up

In this case, the Perfect Magnetic Conductor (PMC) ground plane can be applied to solve the radiation problem because the induced image current in PMC ground plane would have the same direction as the current distribution in the antenna which can boost the gain and radiation efficiency. However, no natural material could be used as PMC structure, so AMC structure is proposed to mimic PMC surface within a specific frequency band by designing periodic structures over a ground plane.

The AMC structure consists of Partial Reflective Surface (PRS) in metal 1 layer and ground plane at the backside of the chip shown in Fig 1. The AMC structure could be optimized to 
obtain the reflection coefficient of $\Gamma=+1$, which indicates inphase reflection at the operating frequency.

\section{DESIGN AND SimULATION: AMC-BASED ANTENNA}

\section{A. AMC Surface}

In this work, the square patch is selected as the AMC unit cell placed on the M1 layer because the square patch AMC structure has a larger bandwidth compared with other AMC structures according to the research of AMC structures comparison [5]. This work defined the operating bandwidth as the frequency band within $+/-45$ degree reflection phase. Fig 2 shows the simulation model of the square patch unit cell, basing on the standard $0.18 \mu \mathrm{m}$ CMOS technology. It contains two PMC and PEC boundary walls in parallel respectively for simulating the infinite AMC surface. A wave-port is assigned on the top boundary and de-embedded to the AMC surface after the simulation.

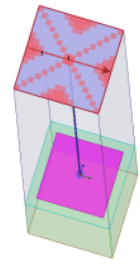

(a)

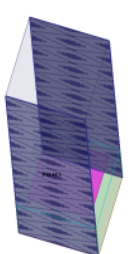

(b)

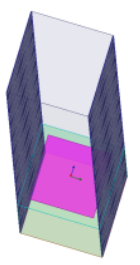

(c)
Fig. 2. HFSS simulation model (a) The AMC unit cell and the wave-port excitation. (b) PEC boundary walls. (c) PMC boundary walls.

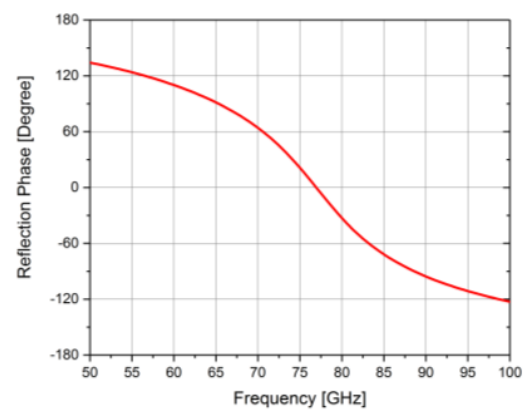

Fig. 3. Reflection Phase of the AMC structure

The dimension of the square patch unit cell is optimized to work at $77 \mathrm{GHz}$. The optimized AMC structure possesses 0 degree reflection phase at $77 \mathrm{GHz}$. The bandwidth of $8.8 \mathrm{GHz}$ is obtained, varying from 72.5 to $81.3 \mathrm{GHz}$.

\section{B. AMC-based On-chip Monopole Antenna}

The simulation model of the AMC-based on-chip monopole antenna is illustrated in Fig 4. It consists of AMC surface with $6 * 8$ unit cells on the M1 layer and a CPW-fed monopole antenna on the M6 layer. Although the higher gain could be obtained by increasing the area of the AMC surface, the AMC size of $6 * 8$ is chosen because of limited chip area. As what is shown in Fig 4, a good impedance matching is obtained at 77 $\mathrm{GHz}$ with a relative bandwidth of $20.8 \%$. Moreover, the gain of $1.7 \mathrm{dBi}$ is achieved at the antenna boresight shown in Fig 5.

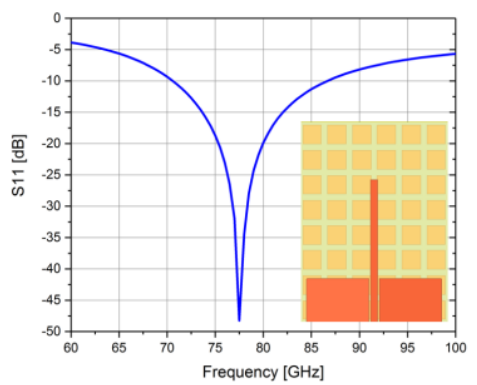

Fig. 4. S11 and top view of the AMC-based monopole antenna
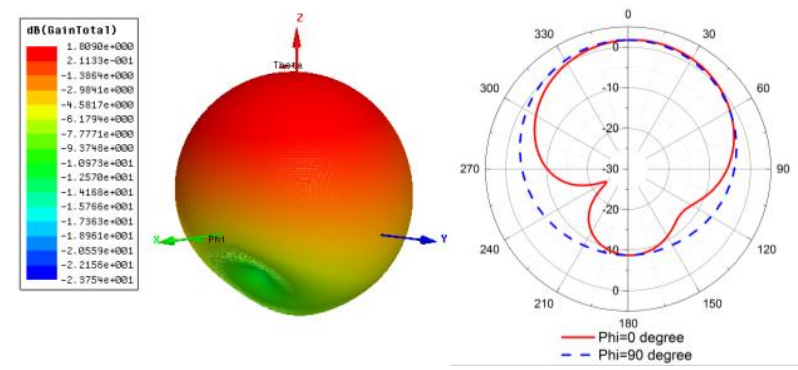

Fig. 5. Radiation Pattern of the AMC-based monopole antenna

Table 1 shows the comparison of the on-chip antenna radiation performance in different cases. The antenna with AMC surface shows an $8.4 \mathrm{~dB}$ gain enhancement compared with standalone antenna and $27.51 \%$ efficiency enhancement compared with an antenna on PEC surface.

TABLE I. RADIATION PERFORMANCE

\begin{tabular}{|c|c|c|c|}
\hline \multirow{2}{*}{} & \multicolumn{3}{|c|}{ CPW-fed On-chip Monopole Antenna } \\
\cline { 2 - 4 } & Standalone & PEC surface & AMC surface \\
\hline Gain & $-6.7 \mathrm{dBi}$ & $-2.3 \mathrm{dBi}$ & $1.7 \mathrm{dBi}$ \\
\hline Efficiency & $32.27 \%$ & $15.01 \%$ & $42.52 \%$ \\
\hline
\end{tabular}

\section{CONCLUSION}

This paper proposed a $77 \mathrm{GHz}$ on-chip monopole antenna with a square patch based AMC surface in $0.18 \mu \mathrm{m}$ CMOS process. The gain and radiation efficiency of $1.7 \mathrm{dBi}$ and $42.52 \%$ are achieved respectively.

\section{REFERENCES}

[1] H. M. Cheema and A. Shamim, "The last barrier: On-chip antennas," IEEE Microw. Mag., vol. 14, no. 1, pp. 79-91, Jan.-Feb. 2013

[2] J. M. Edwards and G. M. Rebeiz, "High-efficiency elliptical slot antennas with quartz superstrates for silicon RFICs," IEEE Trans. Antennas Propag., vol. 60, no. 11, pp. 5010-5020, Nov. 2012.

[3] H. Chu, Y. X. Guo, T. G. Lim, Y. M. Khoo, and X. Shi, "135-Ghz Micromachined On-Chip Antenna and Antenna Array," IEEE Trans. Antennas Propag, vol. 60, no. 10, pp. 4582-4588, Oct 2012.

[4] A. Babakhani, X. Guan, A. Komijani, A. Natarajan, and A. Hajimiri, "A 77-GHz Phased-Array Transceiver With On-Chip Antennas in Silicon: Receiver and Antennas," IEEE Journal of Solid-State Circuits, vol. 41, no. 12, pp. $2795-2806,2006$.

[5] B. S. Cook and A. Shamim, "Utilizing wideband AMC structures for high-gain inkjet-printed antennas on the lossy paper substrate," IEEE Antennas Wirel. Propag. Lett., vol. 12, pp. 76-79, 20 